-1 , max 12); and the median interval between cycles was 14.81 months (6-15.75, $\min 6, \max 120)$.

The EAS included SLE with 23 (43.4\%) cases, systemic sclerosis with 7 (13.2\%) cases, Sjögren's syndrome with $6(11.3 \%)$ cases, vasculitis with $5(9.4 \%)$ cases, Still disease with $3(5.7 \%)$ cases, autoinmune cytopenias with $3(5.7 \%)$ cases, dermatomyositis with 2 (3.8\%) cases, Behçet's disease with 2 (3.8\%) cases, IgG4 disease with $1(1.9 \%)$ case and sarcoidosis with 1 case $(1.9 \%)$

A partial response was observed in 27 patients $(50.9 \%)$ and complete in 20 patients $(37.7 \%)$. There was no response in 6 of the 53 patients $(11.3 \%)$. The response by disease groups is detailed in Table 1 .

\begin{tabular}{lccc}
\hline SAD & Partial response & Complete response & No response \\
\hline SLE $(n=23)$ & $9(39,1 \%)$ & $12(52,2 \%)$ & $2(8,7 \%)$ \\
Systemic Sclerosis $(n=7)$ & $5(71,4 \%)$ & $2(28,6 \%)$ & 0 \\
Vasculitis $(n=5)$ & $5(100 \%)$ & 0 & 0 \\
Sjögren $(n=6)$ & $3(50 \%)$ & $3(50 \%)$ & 0 \\
Al cytopenias $(n=3)$ & $1(33,3 \%)$ & $1(33,3 \%)$ & $1(33,3 \%)$ \\
Dermatomyositis $(n=2)$ & $1(50 \%)$ & $1(50 \%)$ & 0 \\
Behçet's disease $(n=2)$ & 0 & 0 & $2(100 \%)$ \\
lgG4 related disease $(n=1)$ & 0 & $1(100 \%)$ & 0 \\
Sarcoidosis $(n=1)$ & $1(100 \%)$ & 0 & 0 \\
\hline
\end{tabular}

Conclusions: In our study, patients treated with RTX achieved response in $88.7 \%$; similar than some experiences of RTX off-label use.

Remision of the disease occurs in $50 \%$ of the patients.

The best results are observed in SLE, especially in lupus nephritis, and Sjögren disease.

The results in SS are promising due to the limited therapeutic resources for this disease.

References:

[1] Ramos-Casals M, García-Hernández FJ, de Ramón E, Callejas JL, MartínezBerriotxoa A, Pallarés L, et al. Off-label use of rituximab in 196 patients with severe, refractory systemic autoimmune diseases. Clinical and experimental rheumatology 2010; 28 (4): 468-76.

Disclosure of Interest: None declared

DOI: 10.1136/annrheumdis-2017-eular.6402

\section{AB0459 TREATMENT OF SYSTEMIC AUTOIMMUNE DISEASES WITH RITUXIMAB: SAFETY DATA}

M.L. Velloso Feijoo, N. Plaza Aulestia, S. Rodriguez Montero, J.L. Marenco de la Fuente. Rheumatology Unit, Valme University Hospital, Seville, Spain

Background: Systemic autoimmune diseases (SAD) have traditionally been treated with steroids and immunosuppressants, but not all patients respond to this strategy. Rituximab (RTX) has been used in several SAD with favorable efficacy and safety results; there are only reports of isolated clinical experiences of small series of patients. The description of this drug safety data in daily clinical practice may be relevant.

Objectives: To describe the adverse events and the hospital admissions during the treatment of a series of patients with SAD with RTX.

Methods: Demographic data, related to disease and treatment, response and safety variables were included. We use the EULAR definitions of partial response (improvement of at least $50 \%$ of the main manifestations) and complete response (disappearance of the manifestations), because of the heterogeneity of the SAD. Results: We included 53 patients, $90.6 \%$ were women; the mean age at diagnosis was $31.42 \pm 14.33$ years; and the median duration of disease at the onset of RTX $1.99(0-7.5)$ years. Patients received a median of 2 cycles $(1-3 ; \min -1$, max 12$)$; and the median interval between cycles was 14.81 months $(6-15.75$; min 6 , max 120).

The SAD were SLE with 23 cases $(43.4 \%)$, systemic sclerosis with 7 cases $(13.2 \%)$, Sjögren's syndrome with 6 cases $(11.3 \%)$, vasculitis with 5 cases $(9.4 \%)$, Still disease with 3 cases $(5.7 \%)$, autoinmune cytopenias with 3 cases $(5.7 \%)$, dermatomyositis with 2 cases $(3.8 \%)$, Behçet's disease with 2 cases $(3.8 \%)$, IgG4 disease with 1 case $(1.9 \%)$ and sarcoidosis with 1 case $(1.9 \%)$.

A partial response was observed in 27 patients $(50.9 \%)$ and complete in 20 patients $(37.7 \%)$. There was no response in 6 of the 53 patients $(11.3 \%)$.

Adverse events were detected in 15 of the 53 patients $(28.3 \%)$ and 20 were hospitalized $(37.7 \%)$ during the treatment with RTX (Table 1$)$.

\begin{tabular}{lcc}
\hline Infecctions (9) & Disease activity (8) & Others (3) \\
\hline Respiratory: 7 & LES: 8 & Acute coronary syndrome: 1 \\
Pyelonephritis: 1 & & Vertebral fracture: 1 \\
Stomach flu: 1 & & Metrorrhagia: 1 \\
\hline
\end{tabular}

Three patients developed hypogammaglobulinemia, only one of them was associated with recurrent respiratory infections and even hospitalized in one occasion. Febrile neutropenia was detected in 2 patients, and one of them required admission. Three patients were diagnosed with pneumonia and admitted for supportive and antibiotic treatment. Three other patients suffered gastroenteritis requiring admission in one of them. In two cases, low respiratory infections were repeated requiring admission on 7 occasions. There was also recurrent otitis and a severe hypersensitivity reaction.
Conclusions: The most frequent adverse event were the infectious, mainly respiratory tract infections followed by an infusion reaction. No patient developed opportunistic diseases. This findings are similar than observed in other studies on patients with SAD treated with RTX. Infusion reactions are becoming less frequent, due in part to premedication.

We are dealing with a large number of patients with refractory EAS treated with RTX, so the data obtained from this study show an acceptable safety profile.

References:

[1] Ramos-Casals M, García-Hernández FJ, de Ramón E, Callejas JL, MartínezBerriotxoa A, Pallarés L, et al. Off-label use of rituximab in 196 patients with severe, refractory systemic autoimmune diseases. Clinical and experimental rheumatology 2010;28(4):468-76.

Disclosure of Interest: None declared

DOI: 10.1136/annrheumdis-2017-eular.3890

\section{AB0460 USE OF INTRAVENOUS IMMUNOGLOBULIN (IVIG) IN AUTOIMMUNE RHEUMATIC DISEASES: EXPERIENCE IN A THIRD LEVEL MEDICAL CENTER}

M. Pérez Cristóbal ${ }^{1,2}$, R. Ricaño Rocha ${ }^{2}$, G. Horta Baas ${ }^{2}$, L. Barile Fabris ${ }^{2}$, M. Portela Hernández ${ }^{2} .{ }^{1}$ Rheumatology; ${ }^{2}$ Imss, Mexico City, Mexico

Background: IGIV therapy in rheumatology has been used as an alternative treatment for patients with refractory, severe disease, or with contraindication to the use of conventional immunosuppressive therapy as serious infections; it has recently been increased its use to treat multiple autoimmune diseases, however with a limited number of precise indications as there are not enough studies to increase the level of evidence for the administration of IVIG

Objectives: To describe the experience gained with the use of IVIG in autoimmune rheumatic diseases in a third level medical center

Methods: This is an observational, descriptive and retrospective study. We report the use of IVIG in our clinical practice, efficacy and adverse effects. We included consecutive patients with autoimmune rheumatic diseases that received IVIG between 2012 and 2015 . The information was extracted from clinical records Results: We included 35 patients: 19 women, 16 men, 18 with systemic lupus erythematosus (SLE), 15 with autoimmune inflammatory myopathy, 1 with primary Sjögren's syndrome (SSp) and 1 with polyarteritis nodosa (PAN).

The most common indication was active disease associated with severe infection that contraindicated the use of immunosuppressants in 24 patients and in 11 patients refractory activity disease to conventional therapy. The most frequent indications in patients with SLE were: 6 thrombocytopenias, 5 lupus nephritis, 4 pulmonary hemorrhage and 3 neurolupus; of the group of inflammatory myopathy the indications were: 6 dysphagia, 5 respiratory insufficiency and 4 refractory myopathy. In patients with SSp and PAN the indication was peripheral neuropathy. The mean number of IVIG applications was 3.3 (range 1-15). Activity scales were decreased in all patients with IVIG: mean SLEDAI at baseline 15.6 and in the follow up 4.5; in inflammatory myopathies remission was reached in $86 \%$ of the cases. The steroid dose was significantly reduced in most patients in the follow up. Four patients had adverse effects associated with IGIV: 2 with tachycardia and hypertension, one with acute pulmonary edema and one with hemolytic anemia. Conclusions: In our experience, IGIV administration was effective in controlling the activity of the autoimmune rheumatic disease, mainly in patients with concomitant infection, and with a good safety profile.

Discussion: IGIV was an effective alternative treatment in patients with contraindication of conventional treatment or in refractory disease, however it is a high cost treatment and should be used in well selected cases

\section{References:}

[1] Erwin W. Gelfand, M.D. Intravenous Immune Globulin in Autoimmune and Inflammatory Diseases. N Engl J Med 2012;367:2015-25.

[2] Bem Mulhearn I, and lan N. Bruce. Indications for IVIG in rheumatic disease. Rheumatology 2015; 54: 383-391.

Disclosure of Interest: None declared

DOI: 10.1136/annrheumdis-2017-eular.6694

\section{AB0461 BENEFITS OF VITAMIN D IN SLE DEPEND ON THE ORGAN SYSTEM}

M. Petri ${ }^{1}$, L.S. Magder ${ }^{2} .{ }^{1}$ Johns Hopkins University School of Medicine; ${ }^{2}$ Biostatistics, University of Maryland, Baltimore, United States

Background: Low 25-OH Vitamin D is associated with SLE. Both a randomized clinical trial and a longitudinal cohort study proved that supplementation reduced SLE disease activity.

Objectives: We examined whether Vitamin D benefits in SLE are dependent on the organ system.

Methods: Vitamin D and SLEDAI components were measured at each cohort visit starting in 2010 and 16,519 visits of 1,345 different patients were included. The patients were $92 \%$ female, $50 \%$ Caucasian, $41 \%$ African American.

Organ-specific disease activity was defined as a set of binary variables based on SLEDAI. If the patient received any score for any component, then the patient was defined as having that type of activity.

Interestingly, after adjustment for repeated measures and covariates, the relationship between vitamin $\mathrm{D}$ and immunologic disease activity totally disappeared. 